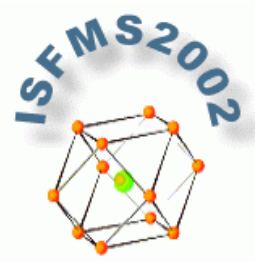

\title{
molecules
}

ISSN 1420-3049

http://www.mdpi.org

\section{A Method for Synthesizing Partially Substituted Cucurbit[n]uril}

\author{
Anthony I. Day*, Alan P. Arnold and Rodney J. Blanch \\ School of Chemistry, University College (UNSW), Australian Defence Force Academy, Canberra ACT \\ 2600, Australia Tel. (61)-2-6268-8089, Fax (61)-2-6268-6268 \\ * Author to whom correspondence should be addressed; e-mail a.day@adfa.edu.au
}

\begin{abstract}
A novel approach to cucurbituril synthesis is described where partial substitution is introduced into cucurbit $[n]$ uril. The identification of homologues (and their substitution) in reaction mixtures is achieved by a combination of ESMS and the use of the molecular probes (guests) 1,4dioxane and 1,9-octanediamine. A unique symmetrical hexamethylcucurbit[3,3]uril, the major product, was isolated and characterized.
\end{abstract}

Keywords: Macrocycles, molecular binding, partially substituted.

\section{Introduction}

The cucurbit $[n]$ uril, $\mathrm{Q}[n]$, are an interesting new family of molecular hosts [1] that are both chemically and thermally robust. They have limited solubility in aqueous solvents, or organic solvents such as formic acid, acetic acid [2], trifluoroacetic acid and trifluoroethanol [3]. For more than ten years decamethylcucurbit[5]uril stood as the only fully substituted cucurbituril [4]. Recently the fully substituted cyclohexanocucurbit[n]uril, $\mathrm{Cy}_{n} \mathrm{Q}[n]$, where $n=5$ and 6, have been reported and these have some organic solvent solubility [5]. While this latest report is encouraging for the possible achievement of other fully substituted cucurbiturils, the limiting factor in the synthesis of fully substituted $\mathrm{Q}[n]$ appears to be that substituted glycolurils have a propensity to form the smaller cucurbituril homologues. In the case of methyl substituted $\mathrm{Q}[n], \mathrm{Me}_{10} \mathrm{Q}[5]$ is the only product formed, whereas for cyclohexano substituted $\mathrm{Q}[n], \mathrm{Cy}_{5} \mathrm{Q}[5]$ occurs as the major product, $>80 \%$. Access to the interesting and potentially more valuable [1] fully substituted higher homologues is restricted. Given that there are only two examples in the literature [4,5] of fully substituted cucurbiturils and despite significant efforts in our laboratories [6,7], the synthesis of various fully substituted $\mathrm{Q}[n]$, bearing the same substitution on every glycoluril unit appears difficult. The smallest homologue consistently forms as the major product when fully substituted Q $[n]$ are successfully synthesized. 
We wish to report a novel approach to cucurbituril synthesis where partial substitution can be introduced into $\mathrm{Q}[n]$. To aid in the discussion of the cucurbiturils of this type, we propose that the original nomenclature [4] be expanded from cucurbit[n]uril, $\mathrm{Q}[n]$, to substituted cucurbit $[s, u]$ uril, $\mathrm{SQ}[s, u]$, to accommodate the substitution, where $s=$ the number of substituted glycolurils and $u=$ the number of unsubstituted glycolurils [6]. The prefix $\mathrm{S}$ describes the substitution carried.
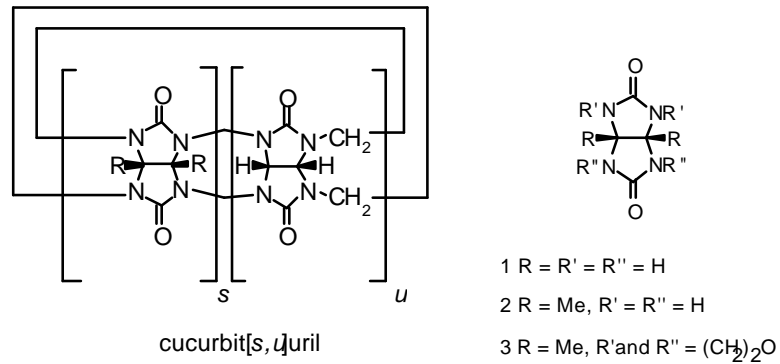

Recently the partially substituted Q[6], diphenylcucurbit[1,5]uril has been synthesized and isolated in moderate yield [8]. Here we describe a method for introducing higher orders of substitution suitable as a model for the development of partially substituted $\mathrm{Q}[n]$ that includes functionality. In addition, chemical manipulation is possible where a range of partially substituted $\mathrm{Q}[n]$ homologues $(n=5-7)$ can be synthesized with the major size being $n=6$. We examined the synthesis of partially substituted $\mathrm{Q}[n]$ building on our previous experiences with the formation of $\mathrm{Q}[n]$ [9] and in particular relying on identification of products by ESMS.

\section{Results and Discussion}

A successful approach was derived from the demonstrated self condensation of dimethyltetracyclic ether (3) in $8 \mathrm{M} \mathrm{HCl}$ which produces the known decamethylcucurbit[5] uril, $\mathrm{Me}_{10} \mathrm{Q}$ [5] [9]. In the formation of $\mathrm{Me}_{10} \mathrm{Q}$ [5] from 3 there are two mole equivalents of methylene linkers more than are required and these are regenerated as formaldehyde. We reasoned that if one mole equivalent of glycoluril (1) were added to $\mathbf{3}$, which carries the linkers, then incorporation of the dimethylglycoluril moiety into the oligomer and subsequent condensation to a partially substituted $\mathrm{Q}[n]$, was probable. By this method, under acid catalysis there are sufficient linkers to achieve $\mathrm{Q}[n]$ of partial substitution without the addition of formaldehyde. ESMS spectra showed the reaction to be successful. We have established that $\mathrm{Q}[n]$ dissolved in a solvent in the presence of $\mathrm{CsCl}$ show ESMS spectra with characteristic patterns of mass ions of the parent ion plus combinations of the alkali metal ions and its counter ion, such as $\left(\mathrm{P}+2 \mathrm{Cs}^{+}\right) / 2, \mathrm{P}+\mathrm{Cs}^{+}$and $\mathrm{P}+\mathrm{Cs}^{+}{ }_{2} \mathrm{Cl}^{-}$[9]. The ESMS spectra of the crude reaction mixture of the above reaction, showed ions attributable to $\mathrm{Q}[n]$ of partial methyl substitution of sizes $n=5-7$, Table 1 . The relative ion intensities of the sizes $(s+u) 5,6$ and 7, were cone voltage dependent and therefore did not accurately reflect the proportions in the reaction mixture. To establish the relative ratios of the sizes, we investigated the mixture by ${ }^{1} \mathrm{H}-\mathrm{NMR}$ spectroscopy (Figure 1a) using two molecular guests as probes. 1,4-Dioxane in $\mathrm{CsCl} / \mathrm{D}_{2} \mathrm{O}$ has previously been used to identify small amounts of $\mathrm{Q}[6]$ in reaction 
mixtures [9] and recently we have found that 1,8-octanediamine in $5 \mathrm{M} \mathrm{DCl} / \mathrm{D}_{2} \mathrm{O}$ can be used to identify Q[7] in reaction mixtures.
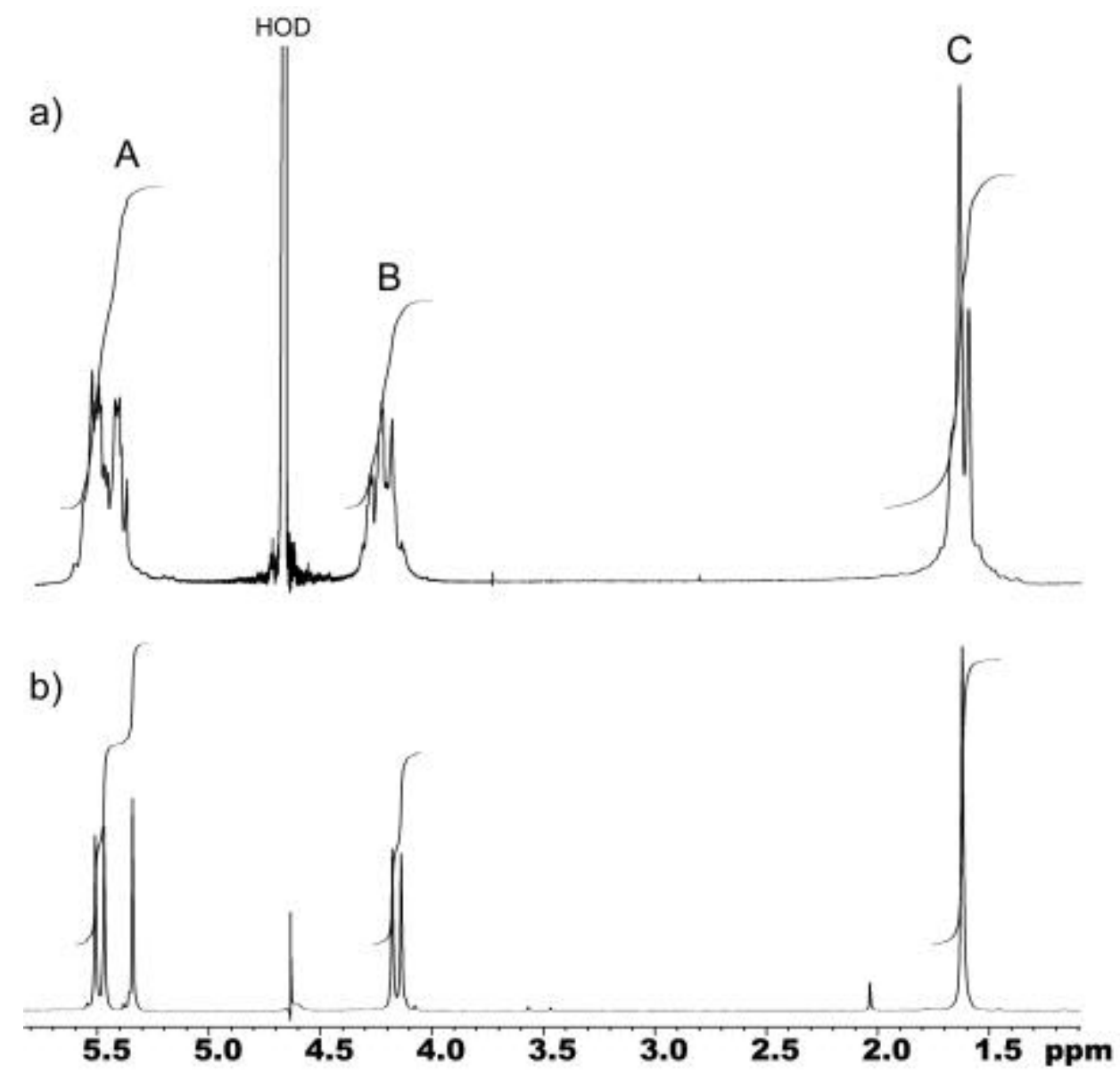

c)

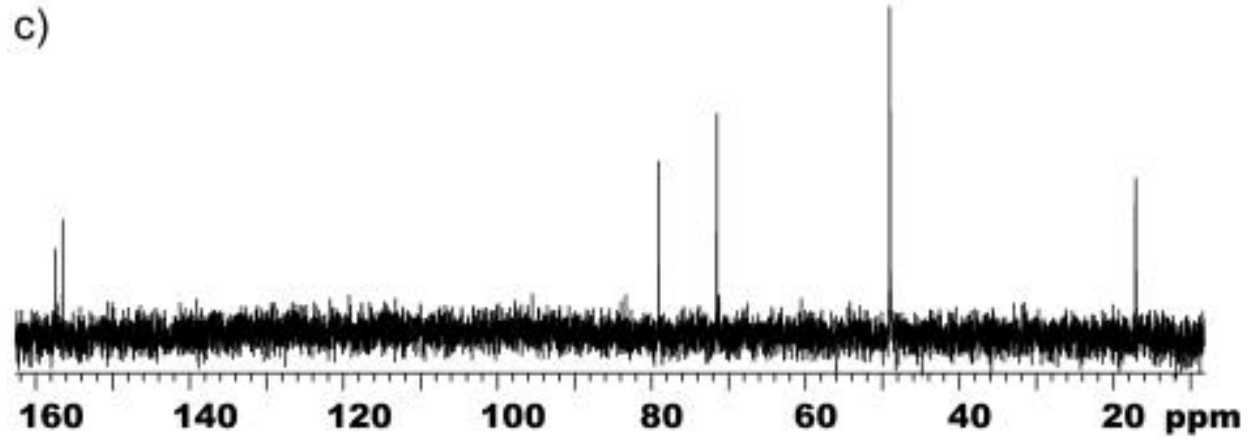

Figure 1. a) The ${ }^{1} \mathrm{H}-\mathrm{NMR}$ spectrum of a sample of the standard crude reaction mixture dissolved in $0.2 \mathrm{M}$ $\mathrm{CsCl} / \mathrm{D}_{2} \mathrm{O}$. The nonequivalent methylene protons occur at $\mathbf{A}$ and $\mathbf{B}$. The methine protons and the methyl protons occur at $\mathbf{A}$ and $\mathbf{C}$, respectively; b) ${ }^{1} \mathrm{H}-\mathrm{NMR}$ spectrum of purified $\mathbf{4 c}$; c) ${ }^{13} \mathrm{C}-\mathrm{NMR}$ spectrum of purified 4c. 
Table 1. ESMS spectral ions observed for the partially substituted cucurbit[s, $u]$ uril at $100 \mathrm{~V}$.

\begin{tabular}{|c|c|c|c|c|c|}
\hline$s+$ & $\mathrm{Me}_{2 s} \mathrm{Q}[s, u]$ & $\%^{\mathrm{a}}$ & $\left(\mathrm{P}+2 \mathrm{Cs}^{+}\right) / 2$ & $\mathrm{P}+\mathrm{Cs}^{+}$ & $\mathrm{P}+\mathrm{Cs}_{2}^{+} \mathrm{Cl}^{-}$ \\
\hline \multirow[t]{4}{*}{5} & $\mathrm{C}_{32} \mathrm{H}_{34} \mathrm{~N}_{20} \mathrm{O}_{10}[1,4]$ & 8 & 562.0 & 990.8 & 1158.9 \\
\hline & $\mathrm{C}_{34} \mathrm{H}_{38} \mathrm{~N}_{20} \mathrm{O}_{10}[2,3]$ & 61 & 576.0 & 1018.9 & 1186.9 \\
\hline & $\mathrm{C}_{36} \mathrm{H}_{42} \mathrm{~N}_{20} \mathrm{O}_{10}[3,2]$ & 100 & 589.8 & 1046.9 & 1214.9 \\
\hline & $\mathrm{C}_{38} \mathrm{H}_{46} \mathrm{~N}_{20} \mathrm{O}_{10}[4,1]$ & 66 & 603.9 & 1075.0 & 1243.0 \\
\hline \multirow[t]{4}{*}{6} & $\mathrm{C}_{40} \mathrm{H}_{44} \mathrm{~N}_{24} \mathrm{O}_{12}[2,4]$ & 22 & 658.9 & 1157.0 & 1324.8 \\
\hline & $\mathrm{C}_{42} \mathrm{H}_{48} \mathrm{~N}_{24} \mathrm{O}_{12}[3,3]$ & 100 & 672.9 & 1185.0 & 1352.8 \\
\hline & $\mathrm{C}_{44} \mathrm{H}_{52} \mathrm{~N}_{24} \mathrm{O}_{12}[4,2]$ & 74 & 686.9 & 1212.9 & 1380.8 \\
\hline & $\mathrm{C}_{46} \mathrm{H}_{56} \mathrm{~N}_{24} \mathrm{O}_{12}[5,1]$ & 7 & 701.0 & 1241.0 & 1408.9 \\
\hline & $\mathrm{C}_{46} \mathrm{H}_{50} \mathrm{~N}_{28} \mathrm{O}_{14}[2,5]$ & 26 & 742.0 & 1350.9 & \\
\hline & $\mathrm{C}_{48} \mathrm{H}_{54} \mathrm{~N}_{28} \mathrm{O}_{14}[3,4]$ & 100 & 756.0 & 1378.8 & \\
\hline & $\mathrm{C}_{50} \mathrm{H}_{58} \mathrm{~N}_{28} \mathrm{O}_{14}[4,3]$ & 43 & 770.2 & 1406.8 & \\
\hline
\end{tabular}

${ }^{a}$ Relative intensities (\%) within each set (eg. $s+u=5$ ). The relative intensity between unit sizes $(s+u)$ 5:6:7 were 90,100 , and $12 \%$. No products were observed that were completely substituted or fully unsubstituted. Ions consistent with $\mathrm{N}_{2}$ associated (or as inclusion complexes) with $\mathrm{Me}_{4} \mathrm{Q}[2,3]$ and $\mathrm{Me}_{6} \mathrm{Q}[3,2]$ where also observed, a phenomenon reported for $\mathrm{Me}_{10} \mathrm{Q}[5]$ [1c].

Significantly, neither of these two molecule guests showed any encapsulation in Q[5] and were therefore considered suitable as probes to identify Q[6]- and Q[7]-sized cavities in the product mixture of the partially substituted Q $[n]$. Dioxane is encapsulated inside the cavity of Q[6] and the slow exchange results in a sharp signal of bound dioxane at $2.71 \mathrm{ppm}$, upfield of free dioxane at $3.56 \mathrm{ppm}$. The upfield shift is characteristic of encapsulation [1a,1d,9]. Unsubstituted Q[7] also binds dioxane but exchanges more quickly giving a broad weak upfield signal between 2.45 and $2.96 \mathrm{ppm}$. Adding dioxane to the crude reaction mixture of methyl substituted cucurbit $[s, u]$ uril, $\mathrm{Me}_{2 s} \mathrm{Q}[s, u]$ in $\mathrm{CsCl} / \mathrm{D}_{2} \mathrm{O}$, produces four sharp signals in the ${ }^{1} \mathrm{H}$-NMR spectrum at 2.61, 2.62, 2.64, and $2.65 \mathrm{ppm}$ (Figure 2a). Given that these signals occurred near the chemical shift of bound dioxane of unsubstituted Q[6] $(2.71 \mathrm{ppm})$, it is reasonable to conclude that the signals are due to dioxane bound in cavities equivalent in size to that of $\mathrm{Q}[6], \mathrm{Me}_{4} \mathrm{Q}[2,4], \mathrm{Me}_{6} \mathrm{Q}[3,3], \mathrm{Me}_{8} \mathrm{Q}[4,2]$, and $\mathrm{Me}_{10} \mathrm{Q}[5,1]$. Since neither $\mathrm{Me}_{12} \mathrm{Q}[6]$ nor Q[6] were detected by ESMS and that the signals were relatively sharp, the observation not only supported the existence of $\mathrm{Me}_{2 s} \mathrm{Q}[s, u]$, as products, where $s+u=6$, but that there were at least four different combinations of substitution, also consistent with the ESMS. 


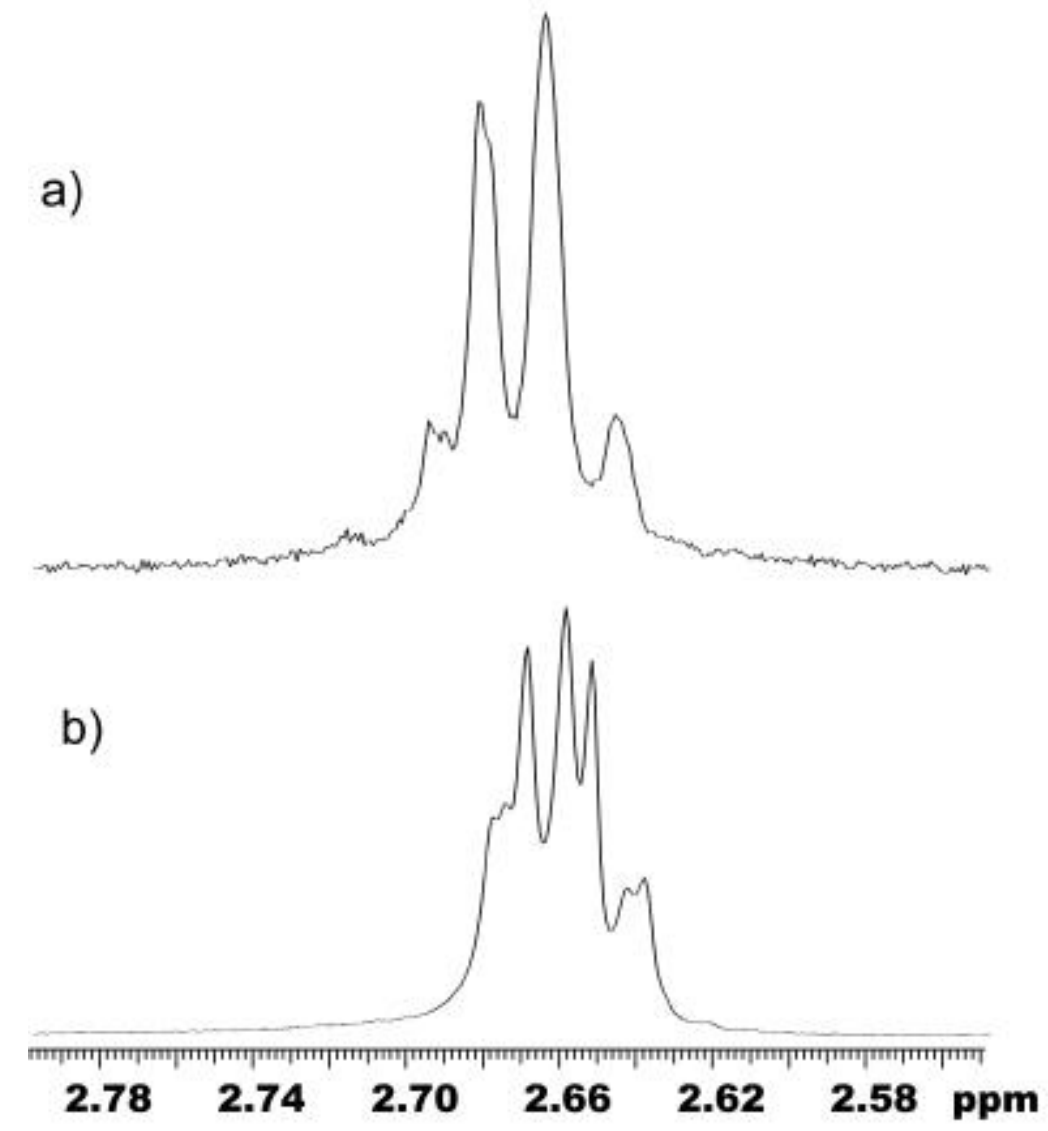

Figure 2. a) An expanded region of the ${ }^{1} \mathrm{H}-\mathrm{NMR}$ spectrum showing the bound dioxane signal of a standard reaction mixture of $\mathrm{Me}_{2 s} \mathrm{Q}[s, u]$ dissolved in $\left.0.2 \mathrm{M} \mathrm{CsCl} / \mathrm{D}_{2} \mathrm{O} ; \mathrm{b}\right)$ An expanded region of the ${ }^{1} \mathrm{H}-\mathrm{NMR}$ spectrum showing the bound dioxane signal of a standard reaction mixture of $\mathrm{Me}_{2 s} \mathrm{Q}[s, u]$ prepared under the concentrated reaction conditions dissolved in $0.2 \mathrm{M} \mathrm{CsCl} / \mathrm{D}_{2} \mathrm{O}$.

By comparing the integral of the bound dioxane signals to the integral of the cucurbituril methylene signals between $4.00-4.28 \mathrm{ppm}$, and assuming that the ratio of the binding of dioxane to $\mathrm{Me}_{2 s} \mathrm{Q}[s, u](s+u=6)$ was comparable to $\mathrm{Q}[6]$, we calculated the proportion of $\mathrm{Q}[6]$-sized cavities $\left(\mathrm{Me}_{4} \mathrm{Q}[2,4], \mathrm{Me}_{6} \mathrm{Q}[3,3]\right.$, $\mathrm{Me}_{8} \mathrm{Q}[4,2]$, and $\left.\mathrm{Me}_{10} \mathrm{Q}[5,1]\right)$. Further details of this procedure are outlined in the Experimental. Repeating the reaction several times gave the proportion of substituted Q[6] as 55 - 60 weight\% of the crude reaction mixture each time.

Using a similar method except with 1,8 -octanediamine in $5 \mathrm{M} \mathrm{DCl} / \mathrm{D}_{2} \mathrm{O}$, the proportion of Q[7]-sized cavities was calculated to be $10-15 \%$ of the total reaction mixture. This was achieved by comparing the integral of 1,8-octanediamine $\alpha$ methylene protons to the integral of the same set of methylene cucurbituril resonances as above. This diamine, in excess, forms a 1:1 complex with unsubstituted Q[7] with slow exchange. Characteristic upfield resonances at $2.55\left(\alpha \mathrm{CH}_{2}\right), 0.77\left(\mathrm{\beta CH}_{2}\right), 0.46$ and $0.38 \mathrm{ppm}$ appear for the 
diamine methylenes when they are inside the cavity. The unbound diamine resonances occur as a set of three peaks at 2.81, 1.47, and $1.13 \mathrm{ppm}$. In contrast, when encapsulated in $\mathrm{Q}[6]$, the $\alpha \mathrm{CH}_{2}$ of this diamine moves downfield [10] as they fall into the shielding zone of the portal, allowing clear distinction. Multiple sets of bound signals as found with dioxane were not observed although some broadening was apparent. The relatively clean ${ }^{1} \mathrm{H}$-NMR spectrum of the crude reaction mixture (Figure 1a) indicates that substitution can be achieved with high conversion to $\mathrm{Me}_{2 s} \mathrm{Q}[s, u]$ while also maintaining a significant proportion of the higher homologues where $s+u=6$ and 7 .
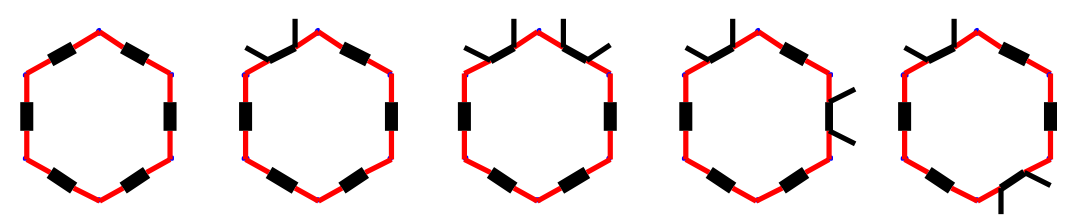

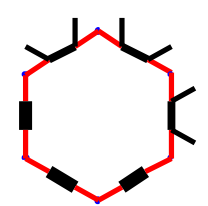

$4 a$
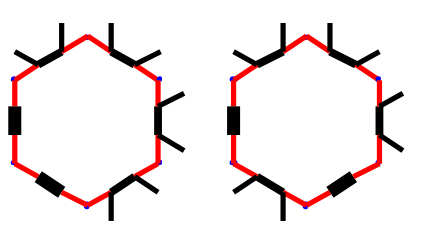

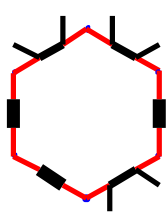

4b

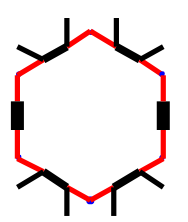

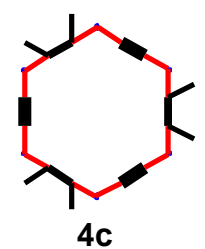

- glycoluril

dimethylglycoluril

$\sim$ methylene link

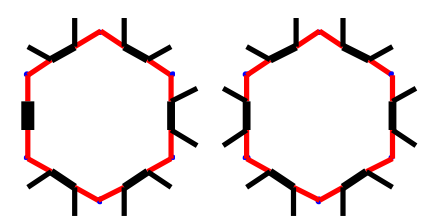

Figure 3. A pictorial representation of the possible substitution patterns to be found in $\mathrm{Me}_{2 s} \mathrm{Q}[s, u]$ where $s+$ $u=6$ and $s=0$ to 6 and $u=0$ to 6 .

If condensation is purely a random process there are ten possible combinations for $\mathrm{Me}_{2 s} \mathrm{Q}[\mathrm{s}, \mathrm{u}]$, where $s+$ $u=6$, within the four basic groups detected by ESMS (Figure 3). It was anticipated however, that the condensation would not be random and that condensation between $\mathbf{1}$ and $\mathbf{3}$ would occur in an alternate manner as shown in Scheme 1. Clearly, two methyl substituted glycolurils can condense adjacent to each other, as indicated by the major ions that identify $\mathrm{Me}_{6} \mathrm{Q}[3,2]$ as a single isomer or a mixture of two possible isomers. In this case the odd number of glycoluril units required to form a Q[5]-sized product precludes the possibility of an alternative mechanism, other than at least two substituted glycolurils condensing adjacent to each other. Given that $\mathrm{Me}_{6} \mathrm{Q}[3,3]$ is comprised of an even number of glycoluril moieties an alternate condensation appeared probable. Chromatography on cation exchange resin and recrystallization of enriched fractions of $\mathrm{Q}[6]$-sized products from $\mathrm{HCl} /$ dioxane gave the complex dioxane @ $\mathrm{Me}_{6} \mathrm{Q}[3,3]$. The dioxane was removed from the complex by repetitive dissolution and evaporation from an aqueous solution of acetonitrile, to yield pure $\mathrm{Me}_{6} \mathrm{Q}[3,3],(\mathbf{4 c})$. The ${ }^{1} \mathrm{H}-\mathrm{NMR}$ spectrum (Figure $1 \mathrm{~b}$ ) in its simplicity shows resonances for a 
single set of non-equivalent methylene protons as doublets at 4.16 and $5.48 \mathrm{ppm}$ and two singlets at 5.35 and $1.62 \mathrm{ppm}$ for the methine and methyl protons respectively. This is consistent with an alternate arrangement of substituted and unsubstituted glycoluril units, hence the symmetrical (about the portal axis) $\mathrm{Me}_{6} \mathrm{Q}[3,3], 4 \mathbf{c}$. The ${ }^{13} \mathrm{C}$-NMR spectrum (Figure 1c) also supports this structure, with two different carbonyl carbon resonances at 157.4 and $156.5 \mathrm{ppm}$, the methyl substituted quaternary carbons at $79.0 \mathrm{ppm}$, the methine carbons at 71.5 ppm, the methylene carbons as a single resonance at $49.0 \mathrm{ppm}$ and the methyl carbon at $17.1 \mathrm{ppm}$. The ESMS spectrum of the $\mathrm{CsCl}$ complexes confirmed $\mathrm{Me}_{6} \mathrm{Q}[3,3]$ was the product, giving three characteristic ions [9] at $m / z$ 673.3, 1213.4 and $1381.2\left(\left(\mathrm{P}+2 \mathrm{Cs}^{+}\right) / 2, \mathrm{P}+\mathrm{Cs}^{+}\right.$and $\mathrm{P}+\mathrm{Cs}^{+}{ }_{2} \mathrm{Cl}^{-}$respectively $)$. A sharp bound dioxane signal was observed at $2.64 \mathrm{ppm}$ corresponding to the major bound dioxane signal observed in the mixture.

\section{Scheme 1}

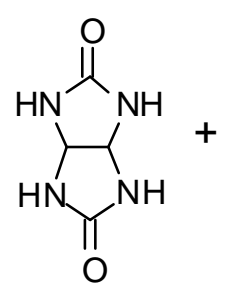

1

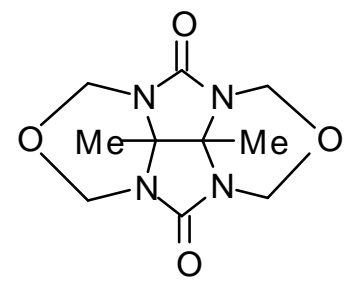

3
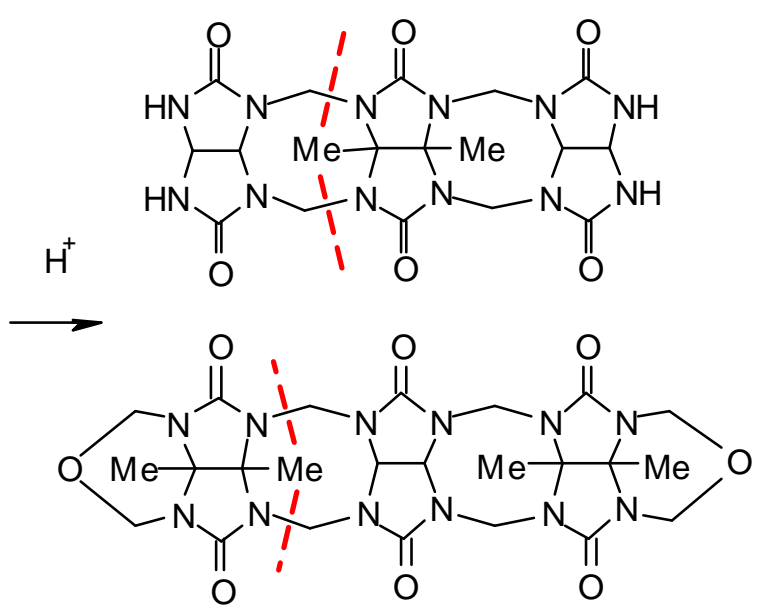

6

Oligomer Alternately Substituted

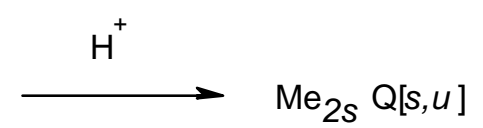

Recently we have developed synthetic methods that can be used to induce a degree of control over the cucurbituril homologue distribution, either by using different acids, by changing reactant concentrations [9], or by adding alkali metal salts [11]. In this work we report preliminary findings that show similar effects on the distribution of partially substituted cucurbituril homologues, $\mathrm{Me}_{2 s} \mathrm{Q}[s, u]$.

$\mathrm{LiCl}$ added to reaction mixtures was found to increase the proportion of $\mathrm{Me}_{2 s} \mathrm{Q}[s, u]$, where $s+u=6$, to $76 \%$, a $20 \%$ improvement. The proportion of $s+u=7$ was virtually unaffected, remaining at $10 \%$, and $s+u$ $=5$ was decreased to $15 \%$. Increasing the concentration of reactants in the absence of $\mathrm{LiCl}$ did not have an anticipated effect of increasing the proportion of higher homologues [9]; rather these conditions had an effect on the number of Me substitution combinations. This was evident in the increased number of bound dioxane 
signals found in the ${ }^{1} \mathrm{H}$ NMR spectrum of the product mixture, 7 signals instead of 4 (Figure $2 b$ ). This result indicates a change in the assemble of glycoluril units during condensation to $\mathrm{Me}_{2 s} \mathrm{Q}[s, u]$. It is probable that under these conditions there is increased opportunity for the dimethylglycoluril moiety or $s$ units, to condense immediately adjacent to each other instead of a repetitive alternate series of condensations of $\mathbf{1}$ and $\mathbf{3}$ (Scheme 1).

$\mathrm{LiCl}$ was found wt only to increase the proportion of Q[6]-sized products but also to affect assembly in favor of the repetitive alternate series of condensations of $\mathbf{1}$ and $\mathbf{3}$. Two changes were evident: the bound dioxane experiment showed an increased signal intensity at $2.64 \mathrm{ppm}$ in the ${ }^{1} \mathrm{H} \mathrm{NMR}$ spectrum, and the proportion of Q[6]-sized product ions was increased relative to other $\mathrm{Q}[n]$ ions in the ESMS spectrum. The proportion of the $\mathrm{Me}_{6} \mathrm{Q}[3,3]$ mass ion also increased (by $10 \%$ ) relative to $\mathrm{Me}_{4} \mathrm{Q}[2,4]$ and $\mathrm{Me}_{8} \mathrm{Q}[4,2](100$ : 33 : 39, respectively, with no other Q[6]-sized combinations detected).

Ideally a technique such as preparative HPLC would be a valuable asset in the isolation of individual components of reaction mixtures in this difficult area of partially substituted $\mathrm{Q}[n]$. Preliminary studies with HPLC indicate that this is possible using a C18 reverse phase column, provided the mixture has been separated into Q[5]-[7] size groups on cation exchange resin prior to HPLC. Solvent gradients of formic acid/water/acetonitrile, of $20 / 80 / 0 \%$ to $5 / 25 / 70 \%$, initially elute the low orders of partially substituted $\mathrm{Q}[n]$. The higher orders of substituted $\mathrm{Q}[n]$ elute with increases in the proportion of acetonitrile. The HPLC fractions were analyzed by ESMS. The major product, the symmetrical $\mathrm{Me}_{6} \mathrm{Q}[3,3], \mathbf{4 c}$, can be isolated by this method, but the yield is difficult to quantify. The HPLC conditions have yet to be optimized and problems regarding detection remain, however the method shows great promise and is likely to be applicable to other substituted and partially substituted Q $[n]$ systems.

The isomers $\mathbf{4 a}$ and $\mathbf{4 b}$ (also of the formula of $\mathrm{Me}_{6} \mathrm{Q}[3,3]$ ), were not detected during the isolation and purification of the symmetrical $\mathrm{Me}_{6} \mathrm{Q}[3,3]$, 4c. The alternate condensation (Scheme 1) is most likely to be controlled by the kinetics of the reaction. The condensation between the methylene carbons of the ether ring of $\mathbf{3}$ (or oligomers) and the urea nitrogen of $\mathbf{1}$ is probably faster than the displacement of the two methylene carbons, a process necessary to allow condensation between two adjacent methyl substituted glycoluril moieties. However, this displacement has been demonstrated by the condensation of $\mathbf{3}$ to form $\mathrm{Me}_{10} \mathrm{Q}$ [5] [9] and in the synthesis of partially substituted $\mathrm{Q}[n]$, by the formation of products such as $\mathrm{Me}_{6} \mathrm{Q}[3,2]$ or $\mathrm{Me}_{8} \mathrm{Q}[4,2]$. Even with these side reaction processes $\mathbf{4 c}$ occurs as the major product with or without $\mathrm{LiCl}$ present. From a $\mathrm{LiCl}$ template reaction [11] the isolated yield of $\mathbf{4 c}$ was $10 \%$. However, the yield in the mixture was $\mathrm{ca} 47 \%$ when calculated by considering the proportion of the integral attributable to bound dioxane in $4 \mathbf{c}(2.64 \mathrm{ppm})$ as a fraction of the percent of Q[6]-sized products. A calculation based on the ratio of the ions of Q[6]-sized products in the ESMS spectrum also gave a similar yield.

Significantly an attempt to synthesize $\mathrm{Me}_{2 s} \mathrm{Q}[\mathrm{s}, \mathrm{u}]$ by direct acid catalyzed condensation of $\mathbf{1}$ and $\mathbf{2}$ in the presence of formaldehyde (as in a conventional $\mathrm{Q}[n]$ synthesis) was less successful; a major portion of the product mixture was unsubstituted $\mathrm{Q}[n]$. This result reflects a competitive reaction where $\mathbf{1}$ reacts with formaldehyde to form an oligomer, and finally $\mathrm{Q}[n]$, which is faster than the incorporation of $\mathbf{2}$ into the oligomer. The favored condensation of $\mathbf{1}$ with itself might also help to explain the formation of products such 
as $\mathrm{Me}_{4} \mathrm{Q}[2,4]$, where at least two unsubstituted glycoluril moieties condense adjacent to each other. This could occur by two pathways, either by regeneration of formaldehyde by hydrolysis of the ether ring of $\mathbf{3}$, or by transfer of a methylene linker to the unsubstituted glycoluril mioety by a hydrolytic disconnection of oligomers. The red dashed lines in Scheme 1 illustrate this type of disconnection for structures 5 and $\mathbf{6}$ [9,12].

$\mathrm{Me}_{6} \mathrm{Q}[3,3]$, (4c), a new type of cucurbituril, was found to be soluble in the polar organic solvents acetonitrile, DMSO and trifluoroethanol, however always requiring the addition of some water. The solubility was $7.5 \mathrm{~g} / 100 \mathrm{~mL}$ (1:1 acetonitrile/water) and similar solubility in trifluoroethanol with the addition of 5-10\% water. This compound is insoluble without the addition of water. It is clear that organic solvent solubility can be achieved even with partial substitution. Organic solvent solubility opens the possibility of a broader range of molecular guest binding capabilities. Cucurbiturils are particularly interesting as unique molecular hosts, with low polarizability [1a].

\section{Conclusions}

Significant insights have been gained in this study toward developing a method for the synthesis of partially substituted cucurbit $[s, u]$ uril, $\mathrm{SQ}[s, u]$, while maintaining the ability to synthesize substantial proportions of the higher homologues $s+u=6$ and 7. In addition a degree of regiochemical control has been achieved. This method for introducing substitution into cucurbiturils gives the potential for solubility manipulation and functionalisation through substituted glycolurils carrying functional groups. Alternative substituted glycoluril combinations are under investigation together with the refinement of HPLC as a purification method for partially $\mathrm{Q}[n]$.

\section{Acknowledgements}

We thank P. Newitt for assistance in the preparation of this manuscript. A.I.D., A.P.A. and R.J.B. acknowledge the support of a URSP grant and a project development fund from Unisearch.

\section{Experimental}

\section{General}

Unless otherwise indicated, commercial materials were used as received. All use of the name dioxane refers to 1,4-dioxane. For ESMS, samples were dissolved in $\mathrm{CsCl} /$ formic acid and diluted with $\mathrm{H}_{2} \mathrm{O}$. ${ }^{1} \mathrm{H}$ and ${ }^{13} \mathrm{C}$-NMR spectra were recorded in $0.2 \mathrm{M} \mathrm{CsCl}$ in $\mathrm{D}_{2} \mathrm{O}$ at 400 and $100 \mathrm{MHz}$ respectively. Chemical shifts are reported in parts per million, relative to external dioxane (3.56 ppm for protons and $67.4 \mathrm{ppm}$ for carbon) in the same solvent. The cation exchange resin used for chromatography was Dowex 50wx2-400 washed with concentrated $\mathrm{HCl}$ then equilibrated with the eluting solvent. 


\section{Procedure}

The tetracyclic diether (3) [13] (1.043gm, 4.04mmol) and the glycoluril (2) (574 mg, $4.04 \mathrm{mmol})$ were combined together and ground into a homogeneous powder. To this we added $\mathrm{LiCl}$ (170 $\mathrm{mg}, 4.0 \mathrm{mmol})$ and $36 \% \mathrm{HCl}(6.5 \mathrm{~mL})$ and the mixture stirred at room temperature for approx $10 \mathrm{~min}$ at which point all solids had dissolved. Heating to $100{ }^{\circ} \mathrm{C}$ for $2 \mathrm{hr}$ resulted in a translucent red-brown solution which was cooled and the acid evaporated in vacuo to give a light brown solid. This was soluble in aqueous salt solutions, TFA and mineral acid solutions. The crude product was dissolved in $0.5 \mathrm{M} \mathrm{HCl} / 90 \%$ formic acid, 1:1 and applied to a column of cation exchange resin. Eluting with the same solvent gave fractions in the order of methyl substituted Q[5], [6] and [7] respectively. Fractions were analyzed by ESMS, and ${ }^{1} \mathrm{H}-\mathrm{NMR}$ spectroscopy using a dioxane probe. Dioxane in $>2$ mole equivalents in $0.2 \mathrm{M} \mathrm{CsCl} / \mathrm{D}_{2} \mathrm{O}$ binds with $\mathrm{Q}[6]$ in an integral ratio of 1:0.5, Q[6]:Dioxane. The fractions containing $\mathrm{Me}_{2 s} \mathrm{Q}[s, u]$ where $s+u=6$ were evaporated in vacuo and the residue dissolved in a small volume of $36 \% \mathrm{HCl}$, followed by the addition of a large excess of dioxane. The colorless crystalline product was collected by filtration, dissolved in acetonitrile/water and evaporated to dryness. This process was repeated 3 times to give $145 \mathrm{mg}$ of $\mathbf{4 c}$, (10\% yield).

\section{References and Notes}

1. (a) Marquez, C.; Nau, W. M. Polarizabilities inside molecular containers. Angew. Chem. Int. Ed. 2001, 40, 4387-4390. (b) Jon, S. Y.; Ko, Y. H.; Park, S. H.; Kim, H.-J.; Kim, K. A facile, stereoselective [2 +2] photoreaction mediated by cucurbit[8]uril. J.C.S. Chem. Commun. 2001, 1938-1939; Park, K.M.; Whang, D.; Lee, E.; Heo, J.; Kim, K. Transition metal ion directed supramolecular assembly of oneand two-dimensional polyrotaxanes incorporating cucurbituril. Chem. Eur. J. 2002, 8, 498-508. (c) Kellersberger, K. A.; Anderson, J. D.; Ward, S. M.; Krakowiak, K. E.; Dearden, D. V. Encapsulation of $\mathrm{N}_{2}, \mathrm{O}_{2}$, methanol, or acetonitrile by decamethylcucurbit[5] uril $\left(\mathrm{NH}_{4}{ }^{+}\right)_{2}$ complexes in the gas phase: Influence of the guest on "lid" tightness. J.Amer.Chem.Soc. 2001, 123, 11316-11317. (d) Kim, J.; Jung, I-S.; Kim, S.-Y.; Lee, E.; Kang, J.-K.; Sakamoto, S.; Yamaguchi, K.; Kim, K. New cucurbituril homologues: Syntheses, isolation, characterization, and X-ray crystal structures of cucurbit $[n]$ uril $(\mathrm{n}=5$, 7, and 8). J. Am. Chem. Soc. 2000, 122, 540-541; Including references found in the following article, Day, A. I.; Blanch, R. J.; Arnold, A. P.; Lorenzo, S.; Lewis G. R.; Dance, I. A cucurbituril-based gyroscane: a new supramolecular form. Angew. Chem. Int. Ed. 2002, 41, 275-277.

2. Jansen, K.; Buschman, H.-J.; Wego, A.; Dopp, D.; Mayer, C.; Drexler, H.-J.; Holdt, H.-J.; Schollmeyer, E. Cucurbit[5]uril, decamethylcucurbit[5]uril and cucurbit[6]uril. Synthesis, solubility and amine complex formation. J. Incl. Phenom. Macrocylic Chem. 2001, 39, 357-363.

3. The cucurbit $[n]$ uril are insoluble in these organic solvents if strictly anhydrous.

4. Flinn, A.; Hough, G. C.; Stoddart, J. F.; Williams, D. J. Decamethylcucurbit[5]uril. Angew. Chem. Int. Ed. 1991, 31, 1475-1477. 
5. Zhao, J.; Kim, H.-J.; Oh, J.; Kim, S.-Y.; Lee, J. W.; Sakamoto, S.; Yamaguchi, K.; Kim, K. Cucurbit $[n]$ uril derivatives soluble in water and organic solvents. Angew. Chem. Int. Ed. 2001, 40, 3155-3160.

6. Day, A. I.; Arnold, A. P.; Blanch, R. J. PCT Int. Appl. 2000-2000AU412 20000505. Priority: AU 99-232 19990507, Unisearch Limited, Australia, WO 2000, p. 112 pp.

7. We have found that pentacyclopentanocucurbit[5]uril, hexacyclopentanocucurbit[6]uril and heptacyclopentanocucurbit[7]uril can be synthesized in conc. $\mathrm{HCl}$ in a ratio of 3.4:2.2:1 respectively, with high conversion from the 3a,4a-cyclopentanoglycoluril.

8. Isobe, H., Sato, S., Nakamura, E., Synthesis of disubstituted cucurbit[6]uril and its rotaxane derivative. Org. Lett., 2002, 4, 1287-1289.

9. Day, A.; Arnold, A. P.; Blanch, R. J.; Snushall, B. Controlling factors in the synthesis of cucurbituril and its homologues. J. Org. Chem. 2001, 66, 8094-8100.

10. Mock, W. L.; Shih, N. Y. Structure and selectivity in host-guest complexes of cucurbituril. J. Org. Chem. 1986, 51, 4440.

11. Day, A. I.; Blanch, R. J.; Coe, A.; Arnold, A. P. The effects of alkali metal cations on product distributions in cucurbit[n]uril synthesis. J .Incl. Phenom.Macrocyclic Chem. 2002, 43, 247-250.

12. Wu, A.; Chakraborty, A.; Witt, D.; Lagona, J.; Damkaci, F.; Ofori, M. A.; Chiles, J. K.; Fettinger, J. C.; Isaacs, L. Methylene-bridged glycoluril dimers: Synthetic methods. J. Org. Chem. 2002, 67, $5817-$ 5830.

13. Niele, F. G. M.; Nolte, R. J. M. Palladium(II) cage compounds based on diphenylglycoluril. J. Am. Chem. Soc., 1988, 110, 172-177.

Sample Availability: Available from the authors.

(C) 2003 by MDPI (http://www.mdpi.org). Reproduction is permitted for noncommercial purposes. 